\title{
BMJ Open The China Patient-centred Evaluative Assessment of Cardiac Events (PEACE) prospective heart failure study design
}

\author{
Xinghe Huang, ${ }^{1}$ Yuan $\mathrm{Yu},{ }^{1} \mathrm{Xi} \mathrm{Li},{ }^{1}$ Fredrick A Masoudi, ${ }^{2}$ John A Spertus, ${ }^{3,4}$ \\ Xiaofang Yan, ${ }^{1}$ Harlan M Krumholz, ${ }^{\oplus, 6,7}$ Lixin Jiang, ${ }^{1}$ Jing $\mathrm{Li}^{1}$
}

To cite: Huang X, Yu Y, Li X, et al. The China Patientcentred Evaluative Assessment of Cardiac Events (PEACE) prospective heart failure study design. BMJ Open 2019;9:e025144. doi:10.1136/ bmjopen-2018-025144

- Prepublication history and additional material for this paper are available online. To view these file, please visit the journa online (http://dx.doi.org/10. 1136/bmjopen-2018-025144)

Received 2 July 2018 Revised 28 November 2018 Accepted 18 December 2018

Check for updates

(C) Author(s) (or their employer(s)) 2019. Re-use permitted under CC BY-NC. No commercial re-use. See rights and permissions. Published by BMJ.

For numbered affiliations see end of article.

Correspondence to

Dr Jing Li; jing.li@fwoxford.org

\section{ABSTRACT}

Introduction China faces the prospect of a large growth in the prevalence of heart failure (HF). However, there is limited knowledge about outcomes in patients after HF hospitalisations, including patient-reported outcomes (PR0s). This paper is to present the study goal, methodology and data collection of the China Patient-centred Evaluative Assessment of Cardiac Events Prospective Heart Failure Study (China PEACE 5p-HF Study).

Methods and analysis The China PEACE 5p-HF Study, a prospective cohort study, will enrol 5000 patients with HF during 2016-2018 from 52 diverse hospitals throughout China and the follow-up period will be 12 months. Information on patients' medical history, in-hospital treatment and in-hospital outcomes are being abstracted from medical records. Details of patients' demographics, socioeconomic status, cardiovascular risk factors, access to healthcare services are being collected through comprehensive baseline interviews. Generic and diseasespecific health status, depression, stress, anxiety and cognitive function are being administered using validated PRO instruments. Follow-up interviews will capture PR0s and hospitalisation events at 1, 6 and 12 months followup. Standardised transthoracic echocardiograms and 6 min walk tests are being done in patients who enrolled in hospitals with these facilities at baseline and at 1 and 12 months after discharge. Collection of blood and urine samples are also being conducted at baseline, 1 and 12 months follow-up and stored for future analyses. Ethics and dissemination The National Center for Cardiovascular Diseases/Fuwai Hospital ethics committee approved this study, and all collaborating hospitals received approval from their local ethics committee. Written informed consent will be obtained from all patients. Findings will be disseminated in future peer-reviewed papers and will help to support improvements in the quality of care for HF nationwide.

Trial registration number NCT02878811.

\section{INTRODUCTION}

The prevalence of heart failure (HF) in low-income and middle-income countries (LMICs) has been poorly characterised. Recent reports show that with the ageing of the population and the epidemiological transition to non-communicable disease, HF

\section{Strengths and limitations of this study}

This study is the first nationwide longitudinal study on clinical outcomes and patient-reported outcomes among patients who are hospitalised primarily for heart failure (HF) in China.

- Comprehensive information included patients' clinical and non-clinical characteristics, in-hospital and long-term care and environmental factors will be collected to predict patients' outcomes and generate risk prediction tools.

- A resource bank for future precision medicine investigations on phenotyping and genotyping of HF will be established, including biosamples, complete in-hospital medical charts, digital/image data of ECG, echocardiogram, $\mathrm{X}$-ray and impedance cardiogram.

- This study will characterise current practice patterns and patients' outcomes in China and help to improve the quality of care nationwide.

is emerging as a major public problem in LMICs, where it constitutes $>2 \%$ of all hospital admissions. ${ }^{12}$ It was estimated in 2000 that there were 4 million patients with chronic HF in China. ${ }^{3}$ As the most populous LMIC, China will be expected to experience a large growth in patients with HF as there are currently an estimated 290 million patients with cardiovascular diseases and its prevalence is rapidly increasing. ${ }^{45}$

In China, as in most countries, little is known about the experience of patients with $\mathrm{HF}$ after hospitalisation. In order to develop strategies to improve recovery and longterm outcomes, it is essential to know about the patient's experience and the factors associated with their outcomes, including patient-reported outcomes (PROs). Data on behaviour, psychological factors, environmental factors, postdischarge management, mortality and readmission have rarely been collected in studies. ${ }^{6-10}$ In addition, few realworld studies, particularly in LMICs, have collected information about PROs, specifically patients' symptoms, function and quality 
of life. Given that a primary therapeutic goal in treating patients with $\mathrm{HF}$ is to improve their health status, the lack of PROs data is an important gap in the current evidence base of HF care in China and throughout the world. ${ }^{11}$ Furthermore, few studies have collected biosamples for biomarker analysis at central laboratories, standardised echocardiograms, ECG or chest X-rays (or chest CT). These data can provide rich information for future investigations on phenotypes and genotypes of $\mathrm{HF}$ to support precision medicine-based efforts to understand the predictors of outcomes and mechanisms of HF, with implications for treatment.

Accordingly, we designed and implemented the China Patient-centred Evaluative Assessment of Cardiac Events Prospective Heart Failure Study (China PEACE 5p-HF Study), which is the first nationwide longitudinal study on both clinical outcomes and PROs among patients who are hospitalised primarily for HF in China. We approached consecutive patients admitted for HF to capture the diverse aetiology of this condition and to 1) examine 1 -year outcomes of patients with HF, including major clinical events (eg, death, hospitalisation for HF, stroke and incident renal insufficiency) and a broad range of PROs (eg, health status, depression and cognitive function); 2) determine predictors of these patient outcomes, including characteristics of patients (eg, demographic, clinical, socioeconomic, psychological, behavioural factors, biomarkers and images), in-hospital and longterm care and environmental factors (eg, air pollution); 3 ) to ultimately generate novel tools for risk prediction among patients with HF and 4) leverage the biospecimens and phenotype data to perform future discovery research. This study will be to identify gaps in evidencebased care as targets for quality improvement in China and provide evidence for improving HF care and formulating the public policy.

\section{METHODS}

\section{Study overview}

The China PEACE 5p-HF Study is based on the China PEACE platform, a collaborative effort among the China National Center for Cardiovascular Diseases (NCCD), the Yale-New Haven Hospital Center for Outcomes Research and Evaluation, the Chinese government and collaborating Chinese hospitals to generate novel knowledge and improve cardiovascular disease outcomes. ${ }^{12}$ This study design was similar to a prospective study of percutaneious coronary intervention (PCI) based on the China PEACE platform as well. ${ }^{13}$ It is a prospective cohort study that will include 5000 patients hospitalised primarily for HF in 52 hospitals (48 are tertiary hospitals and 36 are medical college affiliated hospitals) located in 20 provinces, covering all economic-geographic regions in China (figure 1) (online supplementary material 1). The collaborating hospitals were selected with consideration of their geographical distribution and their capacity to conduct this study (including facilities, staffing, HF

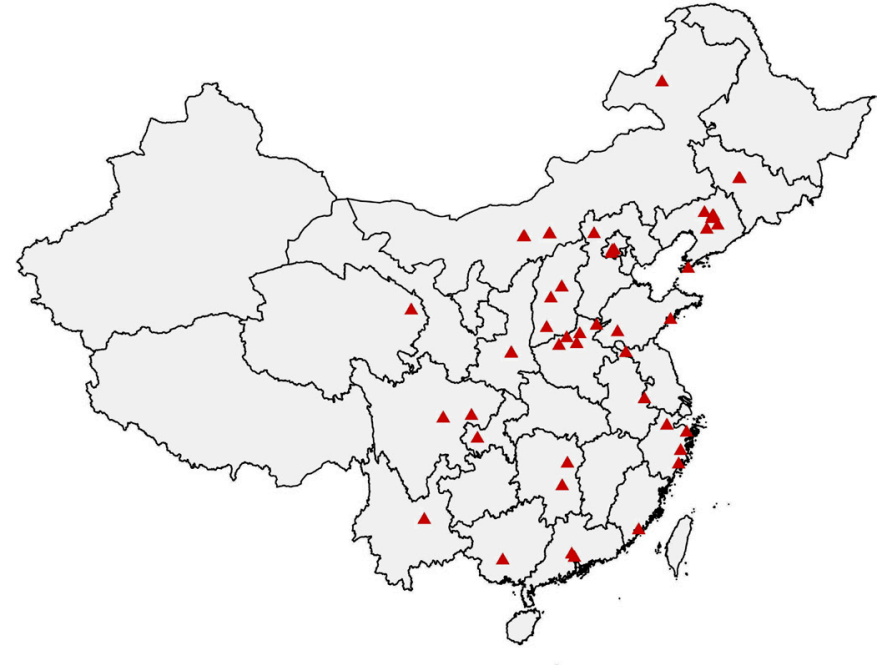

Figure 1 Geographic distribution of participating hospitals in the China Patient- centred Evaluative Assessment of Cardiac Events Prospective Heart Failure Study.

patients volume, etc). Screening and enrolment began in August 2016 and finished in May 2018. Enrolled patients are being interviewed during their index hospitalisation and at 1,6 and 12 months after discharge.

\section{Study population}

Patients hospitalised with a primary diagnosis of new-onset (first diagnosis) HF or decompensation of chronic HF, as assessed by the local physician, are eligible for enrolment. First, trained/certified investigators at each site screen all patients aged 18 years or older admitted primarily for $\mathrm{HF}$, as defined by their admitting physician. If a patient is a local resident and has no difficulty in communication, he/she is invited to participate in the study. To support the generalisability of the findings, the consecutiveness of patient screening is checked against patient databases of local hospitals. Written informed consent is obtained from all enrolled patients before conducting interview (figure 2). To assess potential selection biases, limited data are collected, including demographic, clinical characteristics and in-hospital outcomes, on non-enrolled patients to compare enrolled versus non-enrolled patients.

\section{Data collection}

Data are being collected via patient interview, local examinations and lab tests, central medical chart abstraction and central lab analysis (table 1). We use standardised instruments that have been previously validated for the Chinese population (online supplementary material 2). ${ }^{14-21}$ Local investigators directly enter data into laptop computers equipped with a customised electronic data collection system to allow real-time off-line logic checks to verify the accuracy and completeness of entered data. At the end of each day, the data are transferred to the central secure server at the National Coordinating Center (NCC). To ensure data security, all data are securely stored in an encrypted and password-protected database 


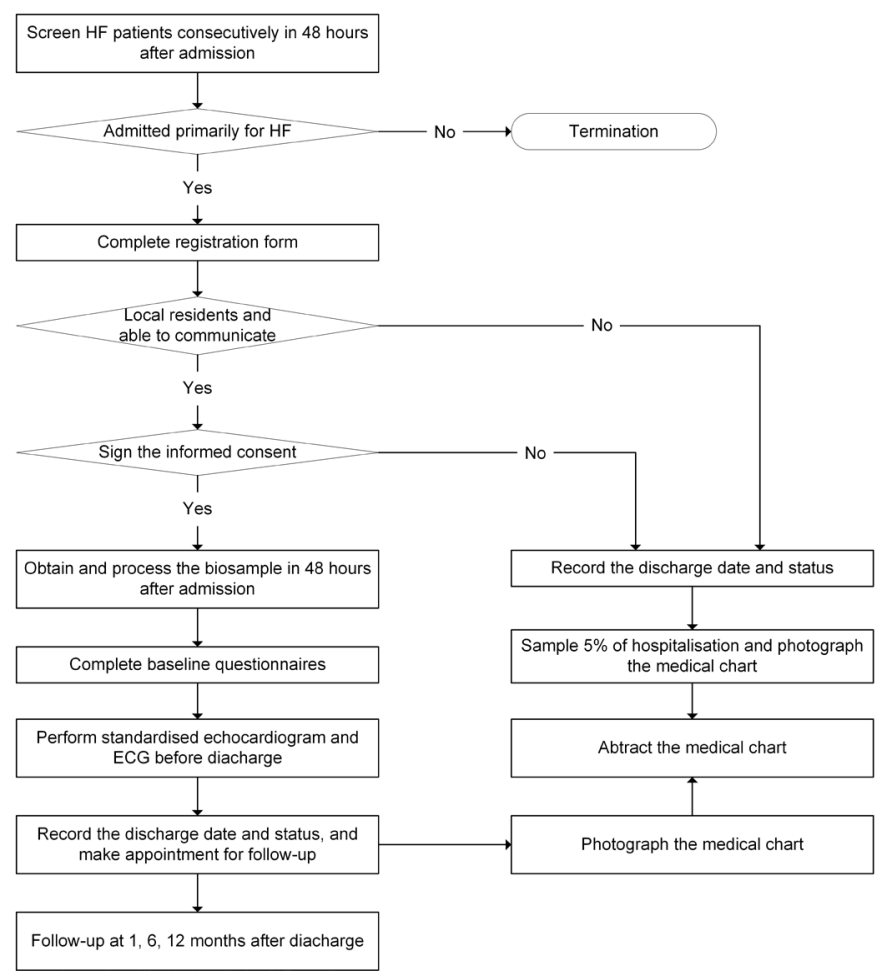

Figure 2 The China Patient-centred Evaluative Assessment of Cardiac Events Prospective Heart Failure Study flow chart. HF, heart failure.

at the NCC with frequent local and off-site backup to secure servers.

\section{Participant interviews}

Participants are being interviewed at baseline (ie, during the index hospitalisation for HF), and at 1, 6 and 12 months following hospital discharge (questionnaires shown in online supplementary material 3). For patients who are not able to attend in-person interviews, central telephone interviews are conducted by the trained investigators at the NCC.

At baseline, we obtain detailed information on demographics, socioeconomic status, cardiovascular risk factors, healthcare service, preadmission medication utilisation and PROs (including generic ${ }^{16}$ and disease-specific health status, ${ }^{1415}$ depression, ${ }^{17}$ stress, ${ }^{18}$ anxiety, ${ }^{19}{ }^{20}$ cognitive function ${ }^{21}$ ) (table 1). PROs measured in this study have undergone linguistic and cultural translations into Mandarin Chinese to ensure validity of the collected data. To measure generic health-related quality of life, the study uses the EuroQol group 5-dimension instrument, which also enables the estimation of country-specific utilities. ${ }^{16}$ We use the short version of the Kansas City Cardiomyopathy Questionnaire to assess HF-specific symptoms, functioning and quality of life.$^{15}$ Psychosocial status is assessed for depressive symptoms (Patient Health Questionnaire (PHQ)-8 for the first 600 enrolled patients and PHQ-2 for the remaining patients), ${ }^{17}$ and cognitive function $(\operatorname{mini}-\mathrm{Cog})^{21}$ in all enrolled patients, while stress (4-item perceived stress scale) ${ }^{18}$ anxiety (2-item Generalised Anxiety Disorder Scale), ${ }^{19}{ }^{20}$ and social support (enhancing recovery in coronary heart disease) ${ }^{22}$ in the first 600 of enrolled patients. At each follow-up, patients are asked to bring in their medications so that a record of all the medications actually being taken can be recorded.

\section{Local examinations and laboratory tests}

Blood pressure, weight and waist circumference are being measured at baseline and each follow-up visit; height and neck circumference are being measured at baseline. For all enrolled patients, a 12-lead ECG is being performed at baseline and at each follow-up visit, local blood cell count is being performed at baseline and at the 1 and 12 months follow-up visits, and chest X-ray (or chest CT) is being performed at baseline. Since not all hospitals can do standardised transthoracic echocardiograms or $6 \mathrm{~min}$ walk tests, these two examinations are only being done in patients enrolled in the hospitals with available facilities at baseline as well as the 1 and 12 months follow-up visits (table 2). In addition, we test participants with an impedance cardiogram (ICG) in seven selected hospitals at baseline and each follow-up visit (online supplementary material 4 ).

To support standardised interpretation of diagnostic cardiac tests, several types of raw image data are collected for future core-lab analysis. These include digital ECG data of 120 s duration, digital chest X-ray (or chest CT) images, echocardiogram static and dynamic images of at least three cardiac cycles (DICOM format), and digital ICG data.

\section{Biosamples analysis at central laboratory and long-term storage}

Blood and urine samples are being obtained at the earliest convenient time after enrolment, typically within 48 hours of hospital admission. Blood and urine samples are also being obtained from all enrolled patients at 1 and 12 months follow-up visit. Samples are being centrifuged, divided into aliquots and frozen within 1 hour following collection by trained site investigators. The samples are being temporarily stored at $-40^{\circ} \mathrm{C}$ or $-80^{\circ} \mathrm{C}$ at local sites, then transported on dry ice to China National Cardiovascular Bio-Bank (Beijing) for central lab analysis (table 1) and long-term storage in liquid nitrogen (online supplementary material 5 ).

\section{Index hospitalisation medical chart abstraction}

For all enrolled patients, we abstract detailed clinical data from an electronic copy of the complete medical record from the index hospitalisation. The chart abstraction is being performed centrally, based on charts with identifying information (name, national ID and contact information) concealed (case report form shown in online supplementary material 6 and data dictionary shown in online supplementary material 7). The part that can be abstracted verbatim without need for interpretation (the face sheet, laboratory test results, echocardiogram report and physician orders) are abstracted via double entry by separate abstractors to ensure accuracy. More 
Table 1 Data collected during the HF index hospitalisation and follow-up

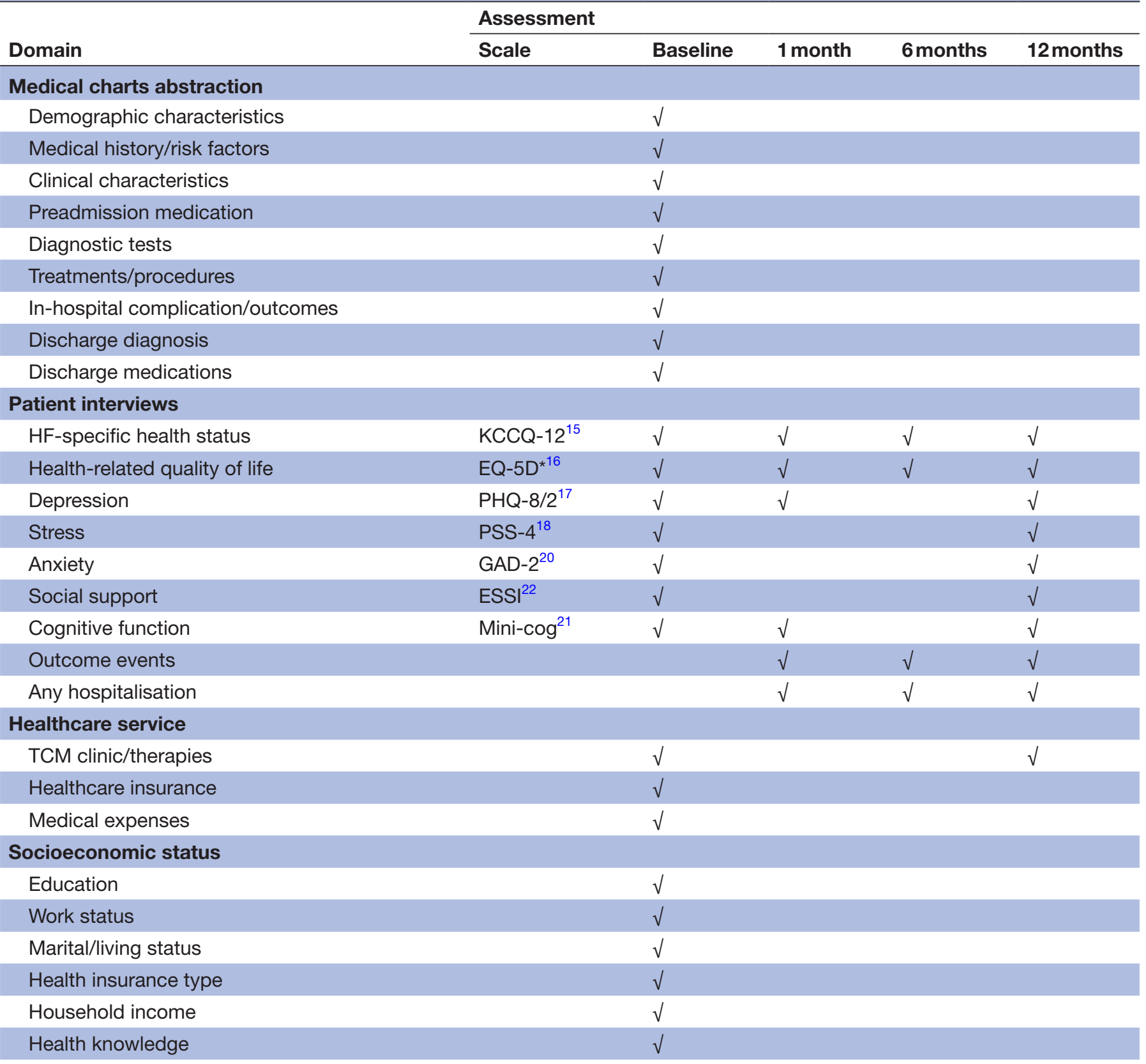

\section{Risk factors}

$\begin{array}{lcc}\text { Smoking status } & \sqrt{ } & \sqrt{ } \\ \text { Lifestyle factors } & \sqrt{ } & \sqrt{ } \\ \text { Physical activity } & \sqrt{ } & \sqrt{ } \\ \text { Alcohol consumption } & \sqrt{ } & \sqrt{ } \\ \text { Obstructive sleep apnoea syndrome } & \sqrt{ }\end{array}$

\section{Medication}

Preventive medications

Side effects of medications

\section{Physical examination}

Blood pressure

Weight

Height

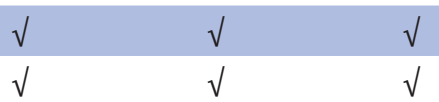

Height 
Table 1 Continued

\begin{tabular}{|c|c|c|c|c|c|}
\hline \multirow[b]{2}{*}{ Domain } & \multicolumn{5}{|c|}{ Assessment } \\
\hline & Scale & Baseline & 1 month & 6 months & 12 months \\
\hline Waist circumference & & $\sqrt{ }$ & $\sqrt{ }$ & $\sqrt{ }$ & $\sqrt{ }$ \\
\hline Neck circumference & & $\sqrt{ }$ & & & \\
\hline Lower extremity oedema & & $\sqrt{ }$ & $\sqrt{ }$ & $\sqrt{ }$ & $\sqrt{ }$ \\
\hline NYHA class & & $\sqrt{ }$ & $\sqrt{ }$ & $\sqrt{ }$ & $\sqrt{ }$ \\
\hline \multicolumn{6}{|l|}{ Local tests } \\
\hline 12-Lead ECG & & $\sqrt{ }$ & $\sqrt{ }$ & $\sqrt{ }$ & $\sqrt{ }$ \\
\hline ICG & & $\sqrt{ }$ & $\sqrt{ }$ & & $\sqrt{ }$ \\
\hline Transthoracic echocardiogram & & $\sqrt{ }$ & $\sqrt{ }$ & & $\sqrt{ }$ \\
\hline 6 min walk test & & $\sqrt{ }$ & $\sqrt{ }$ & $\sqrt{ }$ & $\sqrt{ }$ \\
\hline Blood cell count & & & $\sqrt{ }$ & & $\sqrt{ }$ \\
\hline \multicolumn{6}{|l|}{ Central lab analysis } \\
\hline NT-proBNP & & $\sqrt{ }$ & $\sqrt{ }$ & & $\sqrt{ }$ \\
\hline Troponin & & $\sqrt{ }$ & $\sqrt{ }$ & & $\sqrt{ }$ \\
\hline $\mathrm{HbA1c}$ & & $\sqrt{ }$ & $\sqrt{ }$ & & $\sqrt{ }$ \\
\hline Clinical chemistry & & $\sqrt{ }$ & $\sqrt{ }$ & & $\sqrt{ }$ \\
\hline \multicolumn{6}{|l|}{ Biosamples for long-term storage } \\
\hline Plasma/serum & & $\sqrt{ }$ & $\sqrt{ }$ & & $\sqrt{ }$ \\
\hline DNA & & $\sqrt{ }$ & $\sqrt{ }$ & & $\sqrt{ }$ \\
\hline RNA from periphery blood & & $\sqrt{ }$ & $\sqrt{ }$ & & $\sqrt{ }$ \\
\hline Urine & & $\sqrt{ }$ & $\sqrt{ }$ & & $\sqrt{ }$ \\
\hline
\end{tabular}

*Perform EQ-5D on both the second and seventh day after admission during index hospitalisation.

EQ-5D, EuroQol group 5-dimension self-report questionnaire; ESSI, enhancing recovery in coronary heart disease (ENRICHD) social support inventory; GAD-2, 2-item Generalised Anxiety Disorder Scale; HbA1c, haemoglobin A1c; HF, heart failure; ICG, impedance cardiogram; KCCQ-12, 12-item Kansas City Cardiomyopathy Questionnaire; NT-proBNP, N-terminal pro-B type natriuretic peptide; NYHA, New York Heart Association; PHQ-2/8, Patient Health Questionnaire 2/8 item depression scale; PSS-4, 4-item perceived stress scale; TCM, traditional Chinese medicine.

technical information (the admission record, discharge record, daily record and procedure reports) is abstracted by certified abstractors and reviewed by senior abstractors. Before initiating chart review, each abstractor receives extensive training about the study, orientation to the medical charts and the China PEACE 5p-HF Study data dictionary. We require $>98 \%$ accuracy of abstraction.

\section{Outcome events and adjudication}

We collect patient outcomes after the index hospitalisation, including clinical events and PROs. We collect all hospitalisations during follow-up, with the medical records to support the documentation of outcome events. Clinicians at NCC adjudicate all outcome events including death, resuscitated sudden death, hospitalisation for HF, myocardial infarction, stroke, incident atrial fibrillation and incident renal insufficiency applying criteria employed in clinical trials.

\section{Statistical analysis}

Our strategy of data collection permits a broad range of analyses and analytic approaches, depending on the research question (online supplementary material 8 ). We will calculate summary statistics for outcome events, as well as PROs at baseline and 1, 6 and 12 months after HF. We will report summary statistics for patient demographic, clinical, psychosocial and behavioural characteristics; use of diagnostic tests; treatments received and control of cardiovascular risk factors. To help identify factors associated with outcome events, standard parametric and non-parametric tests for bivariate analyses, including t-test, $\mathrm{X}^{2}$ test, Fisher's exact test and Wilcoxon rank sum test will be used. Furthermore, appropriate multivariable regression analyses, such as linear, logistic, Cox proportional hazard and Poisson models, will be conducted to determine a factor's association with the outcome measures while adjusting for potential confounders. As patients are clustered within hospitals and there are repeated observations clustered within patients, our analyses will account for clustering in data (eg, generalised estimating equations or random effects models) and the use of appropriate repeated measures analyses. Of particular interest will be the variability in patterns of care and their association with outcomes. While all efforts are made to obtain high response rates in follow-up PROs, 
Table 2 Echocardiogram image and measurements

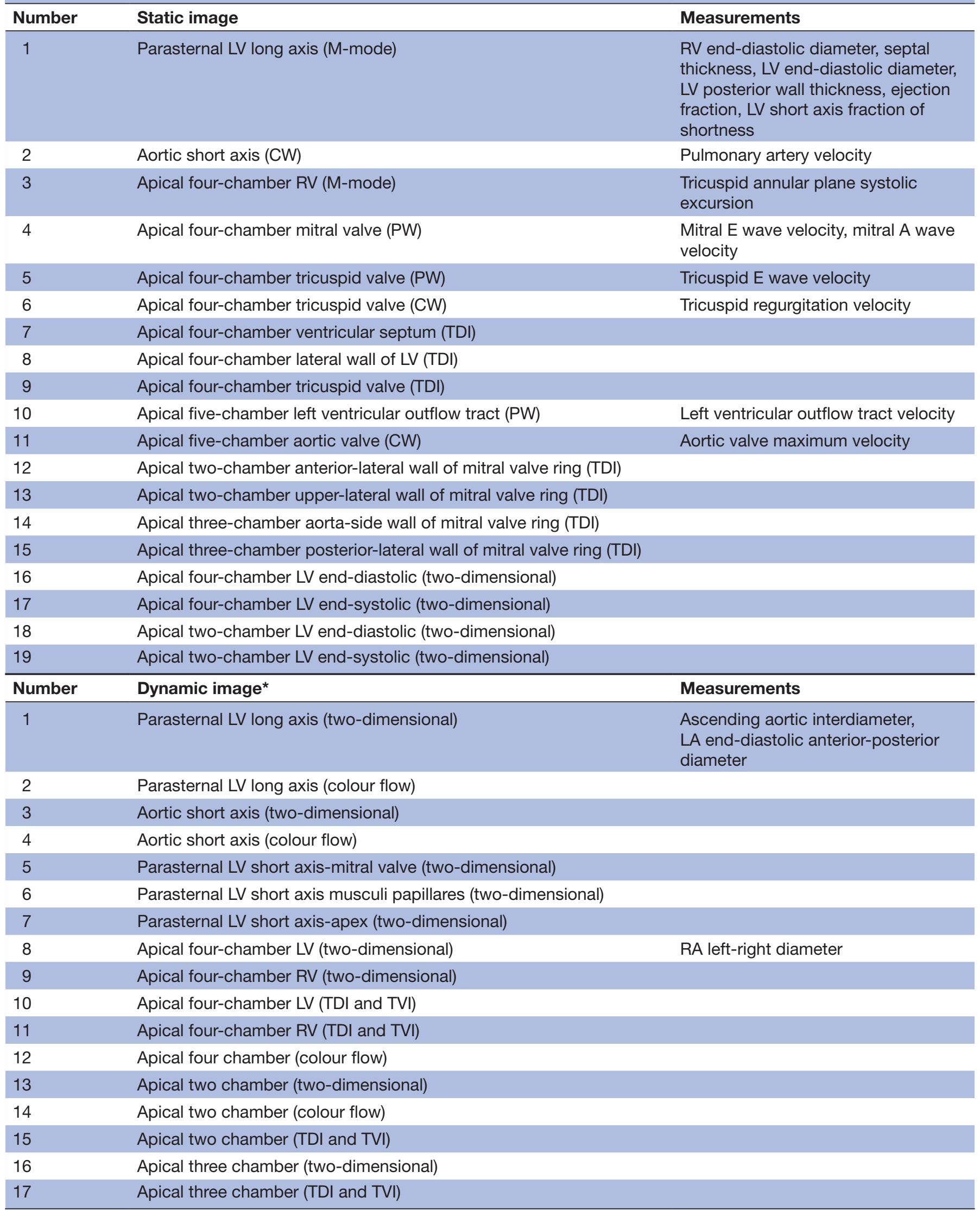


Table 2 Continued

\begin{tabular}{lll}
\hline Number & Dynamic image* & Measurements
\end{tabular}

${ }^{*}$ Collecting image of three to five cardiac cycles.

CW, continuous Doppler; LA, left atrium; LV, left ventricular; PW, pulsed-wave Doppler; RA, right atrium; RV, right ventricular; TDI, tissue Doppler imaging; TVI, tissue velocity imaging.

some missing data are inevitable. We will carefully evaluate any potential selection biases introduced by missing data and conduct inverse probability weighting, when appropriate, based on a propensity model for participation in the follow-up assessments to preferentially weigh the experiences of patients who were most like those who did not participate in follow-up.

The current study is primarily descriptive. The sample size was calculated based on the desired precision of estimates for key outcomes. A sample size of 5000 was determined based on both feasibility and consideration of adequate statistical precision for describing 12-month mortality and PROs in the overall sample (online supplementary material 9).

\section{Ethics and dissemination}

The NCC of this study is based at the NCCD/Fuwai Hospital. All enrolled participants sign an informed consent, including consent for the biosample analysis at central laboratories and long-term storage. Findings will be disseminated in future peer-reviewed articles and will help to support improvements in the quality of care for HF nationwide.

\section{Patient and public involvement}

Patients and public were not involved in the development of the research questions, recruit, design or conduct of the study. Results will be disseminated to patients and public through media, including social media.

\section{DISCUSSION}

The China PEACE 5p-HF Study is the first national longitudinal study that will characterise the care and collect both clinical outcomes and PROs from a diverse population of patients with HF in China. The goal of this study is to assess the broad range of patient outcomes and the variability in management of HF. It will examine the aetiology, subtype and the natural history of HF in China. It will also support comparative effectiveness studies of pharmaceutical and non-pharmaceutical therapies among patients with HF (table 3). Several unique features of this study, including the consecutive screening of patients, the standardised data collection, the collection of patient-centred data elements through serial interviews, the central analysis of biosamples, the collection of dynamic image data of ECG and echocardiogram, the longitudinal follow-up and the central adjudication for clinical events augment the value of this study. In addition, since the China PEACE $5 p-H F$ Study belongs to the national studies of major chronic disease (including cardiovascular disease, stroke, diabetes and chronic kidney disease), the variables have similar data definitions across studies of other diseases and enable analyses that pooling data across studies of other chronic diseases. To our knowledge, this is the first time a study has adopted unified definition across major chronic diseases.

Findings from the China PEACE 5p-HF Study will provide information about the long-term outcomes of patients with $\mathrm{HF}$ and will identify generalisable strategies to improve care and reduce disparities with a focus on a broad range of outcomes, including those that reflect the patient's experience. Within 1 year of a hospitalisation for HF, the mortality rates in Europe and the USA were 17\% and $22 \%$, respectively. The rehospitalisation rate within 1 year in Europe was $44 \%$ and $>50 \%$ patients were readmitted to the hospital within 6 months after discharge in the USA. ${ }^{23-25}$ There have been few studies in China to report the long-term outcomes of patients with HF until this study. In addition, PRO measures will provide critically important, and currently unknown, information regarding the burden of HF in China from the patients' perspective. The comprehensive understanding of the longitudinal course of HF will support quality improvement initiatives and key public policies for HF in China, as well as illuminate the efforts in other LMICs facing similar challenges.

The standardised collection of comprehensive data will facilitate an understanding of factors influencing patient outcomes and generate risk prediction tools. In China PEACE 5p-HF Study, we collect patient characteristics (eg, demographic, clinical, social economical, psychosocial and behavioural factors), in-hospital and posthospital cares as well as biosamples for central laboratory analysis, 120 s ECG with uniform equipment and echocardiograms with standard protocol. This will enable investigators to define the prognostic importance of individual factors on long-term mortality, admission to hospital and impaired quality of life.

This study will also examine the in-hospital care and postdischarge long-term management, thus enabling the evaluation of the comparative effectiveness of HF therapies among Chinese patients with HF, examine the associations of variability in care with outcomes and identify targets for quality improvement. Data from this study will be used to assess clinical and non-clinical factors associated with the use of preventative interventional procedures, as well as the association between long-term management and patient outcomes to address a substantial gap in knowledge regarding long-term management for HF in China. As most prior studies focused only on 
Table 3 The themes, resources and data collected and strength of the China PEACE 5p-HF Study

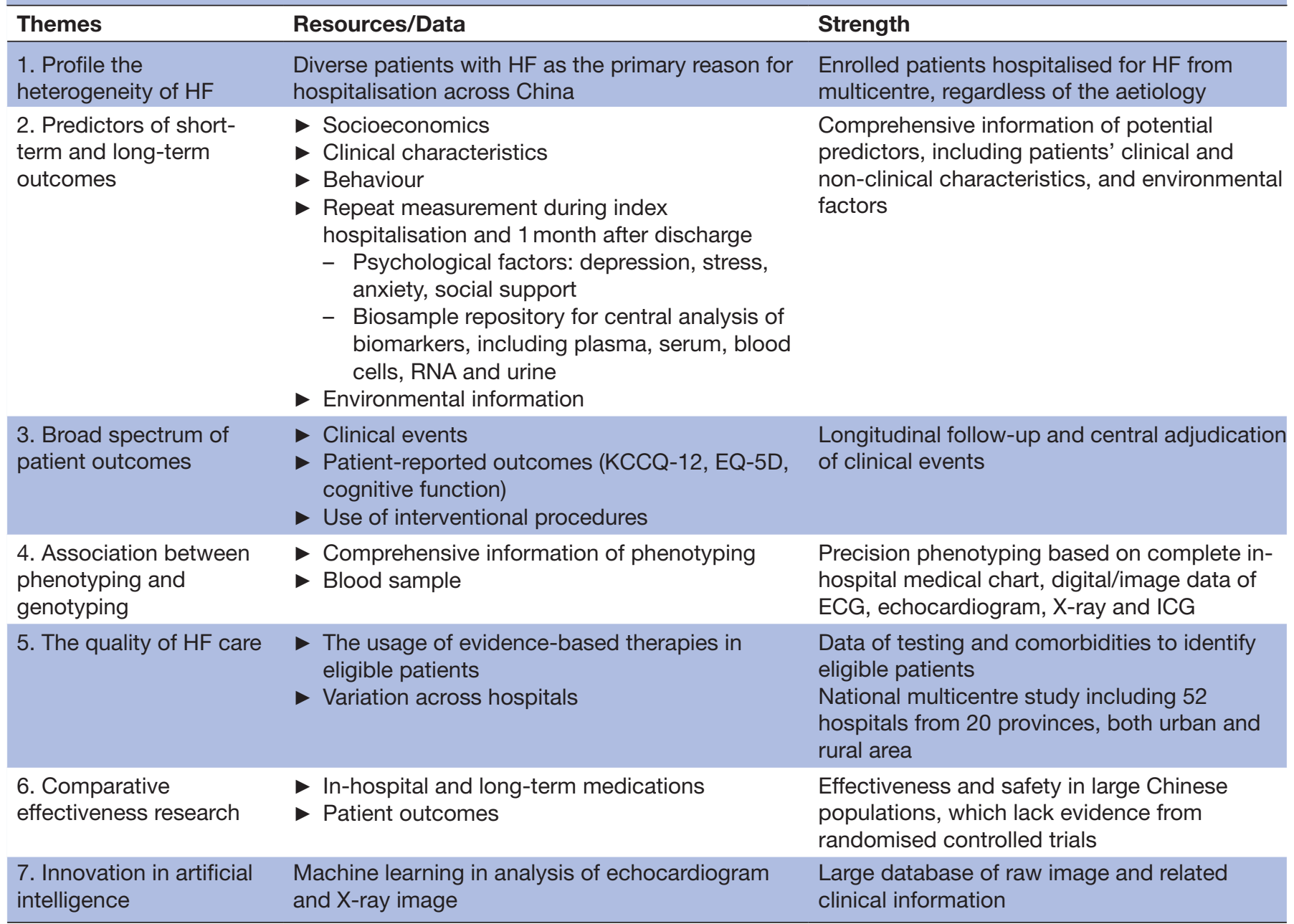

China PEACE 5p-HF Study, China Patient-centred Evaluative Assessment of Cardiac Events Prospective Heart Failure Study; EQ-5D, EQ5D, EuroQol group 5-dimension self-report questionnaire; HF, heart failure; ICG, impedance cardiogram; KCCQ-12, 12-item Kansas City Cardiomyopathy Questionnaire.

in-hospital care and discharge medications, ${ }^{79}{ }^{10}$ there is a continued need to understand the long-term medications, the maintained dosage as well as associated patient characteristics and institutional factors.

HF is a common manifestation of diverse cardiovascular diseases and the patient population is heterogeneous. Heart failure with preserved ejection fraction (HFpEF) and heart failure with reduced ejection fraction (HFrEF) seem to have different epidemiological and aetiological profiles and the characteristics of patients with the newly defined heart failure with mid-range ejection fraction are between those with HFrEF and HFpEF. ${ }^{26}$ This study will establish a resource bank for future deep investigations on phenotypes and genotypes of HF. Large samples of well-characterised patients will be stored long term as a biorepository, enabling identification of novel biomarkers and genetic loci. In addition, the standardised ECG and echocardiographic data will enable future core lab analyses for more precise phenotypic descriptions of patients with $\mathrm{HF}$ and provide rich information to support precision medicine and mechanistic insights into $\mathrm{HF}$ and its progression.

The strengths of conducting a longitudinal study on clinical outcomes and PROs among patients with HF have been well recognised, which help to better understand where the greatest opportunities lie in optimising patient outcomes in the face of an increasing burden. In addition, the established organisational infrastructure of our research team, integrating resources from the Chinese government, a diverse hospital network and an international research team ensure a rigorous study design and rapid dissemination of findings. Furthermore, the implementation of a high-quality and cost-effective study is supported by a broad research network, as well as a robust management system. During the past decade, China NCCD has collaborated with some of the world's leading academic institutions to conduct several of the largest clinical trials in China. ${ }^{27-30}$ In-depth training and rigorous quality control procedures are being conducted continuously to maintain the robust research network. 
We have also established a management system for large multicentre studies, which has internationally accepted quality standards and be capable of supporting research work in diverse healthcare settings in China. The collaborative research and performance improvement network created by the China PEACE platform will ultimately improve patient outcomes and may present a model for research and quality improvement in other international settings.

It should be noted that only patients who consent are enrolled and followed-up and these patients may differ from those who are eligible but do not consent to participate. However, it is a common problem faced by all observational studies and we plan to evaluate potential selection biases by collecting information on baseline demographic and clinical characteristics and in-hospital outcomes for non-enrolled patients. Second, it is expected that not all enrolled patients will participate in in-person interview, even though efforts will be made to obtain a high yield, thus we will miss some information. For patients who do not attend face-to-face interviews, central telephone interviews will be conducted by the trained investigators at the NCC to collect other information.

The China PEACE 5p-HF Study is designed to help improve the quality of care in routine practice and patient clinical outcomes, as well as PROs for China and similar LMICs by generating a novel, high-quality and comprehensive data about longitudinal patient outcomes following hospitalisations for HF. This study is also intended to support discovery research. The partnership among the Chinese government, a large network of hospitals with geographic and capability diversity and an international outcomes research team will be leveraged to create a platform for cardiovascular diseases research, which will facilitate policy-making and inform the development of novel quality improvement tools.

\section{Author affiliations}

${ }^{1}$ National Clinical Research Center of Cardiovascular Diseases, State Key Laboratory of Cardiovascular Disease, Fuwai Hospital, National Center for Cardiovascular Diseases, Chinese Academy of Medical Sciences and Peking Union Medical College, Beijing, China

${ }^{2}$ Division of Cardiology, University of Colorado at Denver-Anschutz Medical Campus, Aurora, Colorado, USA

${ }^{3}$ School of Medicine, University of Missouri, Kansas City, Missouri, USA

${ }^{4}$ Saint Luke's Mid America Heart Institute, Kansas City, Missouri, USA

${ }^{5}$ Center for Outcomes Research and Evaluation, Yale-New Haven Hospital, New Haven, Connecticut, USA

${ }^{6}$ Department of Health Policy and Management, Yale School of Public Health, New Haven, Connecticut, USA

${ }^{7}$ Section of Cardiovascular Medicine, Robert Wood Johnson Clinical Scholars Program, Department of Internal Medicine, Yale University School of Medicine, New Haven, Connecticut, USA

Acknowledgements The authors would like to appreciate the multiple contributions made by project teams at the China Oxford Center for International Health Research and Yale New Haven Hospital Center for Outcomes Research and Evaluation in the realms of study design and operations. The authors would like to thank Professor Xuyu Jin from University of Oxford and Professor Christiane E Angermann from Universitätsklinikum Würzburg for their advice on study design. The authors would like to thank Shiwani Mahajan for help in language editing and Ziyue Gao for plotting Figure 1. The authors are also grateful for the support provided by the Chinese government.

Contributors JL, HMK, LJ designed the study and take responsibility for all aspects of it. XH and JL wrote the first draft of the article, with further contribution from YY, XL, FAM, JAS, XY. All authors approved the final version of the article.

Funding This work was supported by the National Key Research and Development Program (2017YFC1310803, 2017YFC1310801) from the Ministry of Science and Technology of China; the CAMS Innovation Fund for Medical Science (2017-I2M-2002, 2017-I2M-B\&R-02, 2016-I2M-1-006, 2016-I2M-2-004); the 111 Project from the Ministry of Education of China (B16005).

Competing interests HMK works under contract with the Centers for Medicare and Medicaid Services to develop and maintain performance measures, is chair of a cardiac scientific advisory board for United Health and is the recipient of research grants from Medtronic and Johnson \& Johnson through Yale University. FAM has a contract with the American College of Cardiology for his role as the Chief Science Officer of the NCDR. JAS has an analytic contract with the American College of Cardiology, performs consultative services for United Healthcare, Janssen, AstraZeneca, Bayer and Novartis and owns the copyright to the Kansas City Cardiomyopathy Questionnaire.

Patient consent for publication Obtained.

Ethics approval The National Center for Cardiovascular Diseases (NCCD)/Fuwai Hospital ethics committee approved this study, and all collaborating hospitals received approval from their local ethics committee.

Provenance and peer review Not commissioned; externally peer reviewed.

Open access This is an open access article distributed in accordance with the Creative Commons Attribution Non Commercial (CC BY-NC 4.0) license, which permits others to distribute, remix, adapt, build upon this work non-commercially, and license their derivative works on different terms, provided the original work is properly cited, appropriate credit is given, any changes made indicated, and the use is non-commercial. See: http://creativecommons.org/licenses/by-nc/4.0/.

\section{REFERENCES}

1. Callender T, Woodward M, Roth G, et al. Heart failure care in low- and middle-income countries: a systematic review and metaanalysis. PLoS Med 2014;11:e1001699.

2. Lam CS, Teng TK, Tay WT, et al. Regional and ethnic differences among patients with heart failure in Asia: the Asian sudden cardiac death in heart failure registry. Eur Heart J 2016;37:3141-53.

3. National Center for Cardiovascular Diseases. Report on cardiovascular diseases in China: Encyclopedia of China Publishing House, 2017.

4. Yang G, Kong L, Zhao W, et al. Emergence of chronic noncommunicable diseases in China. The Lancet 2008;372:1697-705.

5. Lozano R, Naghavi M, Foreman K, et al. Global and regional mortality from 235 causes of death for 20 age groups in 1990 and 2010: a systematic analysis for the Global Burden of Disease Study 2010. Lancet 2012;380:2095-128.

6. Association C M. Retrospective investigation of hospitalized patients with heart failure in some parts of China in 1980, 1990 and 2000. Chinese Journal of Cardiology 2002;30:450-4.

7. Liu X, Yu H, Pei J, et al. Clinical characteristics and long-term prognosis in patients with chronic heart failure and reduced ejection fraction in China. Heart Lung Circ 2014;23:818-26.

8. Liu C, Hou Y, Wang X, et al. Clinical assessment of Shenfu injection loading in the treatment of patients with exacerbation of chronic heart failure due to coronary heart disease: study protocol for a randomized controlled trial. Trials 2015;16:222.

9. Fu R, Xiang J, Bao H, et al. Association between process indicators and in-hospital mortality among patients with chronic heart failure in China. Eur J Public Health 2015;25:373-8.

10. Sun XQ, Liu LS, Lang YJ, et al. Retrospective analysis of hospitalized elderly patients with chronic heart failure in rural parts. Chinese Journal of Gerontology 2009;29:3003-5.

11. Spertus JA. Evolving applications for patient-centered health status measures. Circulation 2008;118:2103-10.

12. Li J, Dreyer RP, Li X, et al. China patient-centered evaluative assessment of cardiac events prospective study of acute myocardial infarction: study design. Chin Med J 2016;129:72-80.

13. Du X, Pi Y, Dreyer RP, et al. The china patient-centered evaluative assessment of cardiac events (PEACE) prospective study of percutaneous coronary intervention: Study design. Catheter Cardiovasc Interv 2016;88:E212-E221. 
14. Deng YH, Dong YG, Chen DD, et al. Evaluation of Kansas City questionnaire of cardiomyopathy in clinical practice in patients with chronic heart failure. Chinese Journal of Cardiology 2004;32:676-9.

15. Spertus JA, Jones PG. Development and validation of a short version of the Kansas City cardiomyopathy questionnaire. Circ Cardiovasc Qual Outcomes 2015;8:469-76.

16. Wang HM, Patrick DL, Edwards TC, et al. Validation of the EQ$5 \mathrm{D}$ in a general population sample in urban China. Qual Life Res 2012;21:155-60.

17. Liu ZW, Yu Y, Hu M, et al. PHQ-9 and PHQ-2 for screening depression in chinese rural elderly. PLoS One 2016;11:e0151042.

18. Leung DY, Lam TH, Chan SS. Three versions of Perceived Stress Scale: validation in a sample of Chinese cardiac patients who smoke. BMC Public Health 2010;10:513.

19. Tong $X, A n D, M c G o n i g a l ~ A$, et al. Validation of the Generalized Anxiety Disorder-7 (GAD-7) among Chinese people with epilepsy. Epilepsy Res 2016;120:31-6.

20. Wild B, Eckl A, Herzog W, et al. Assessing generalized anxiety disorder in elderly people using the GAD-7 and GAD-2 scales: results of a validation study. Am J Geriatr Psychiatry 2014;22:1029-38.

21. Yang L, Yan J, Jin X, et al. Screening for dementia in older adults: comparison of Mini-Mental State Examination, Mini-Cog, Clock Drawing Test and AD8. PLoS One 2016;11:e0168949.

22. Enhancing Recovery in Coronary Heart Disease Patients (ENRICHD): Study design and methods. Am Heart J 2000;139:1-9.
23. Desai AS, Stevenson LW. Rehospitalization for heart failure: predict or prevent? Circulation 2012;126:501-6.

24. Loehr LR, Rosamond WD, Chang PP, et al. Heart failure incidence and survival (from the Atherosclerosis Risk in Communities study). Am J Cardiol 2008;101:1016-22.

25. Maggioni AP, Dahlström U, Filippatos G, et al. EURObservational Research Programme: regional differences and 1-year follow-up results of the Heart Failure Pilot Survey (ESC-HF Pilot). Eur $J$ Heart Fail 2013;15:808-17.

26. Lam CS, Solomon SD. The middle child in heart failure: heart failure with mid-range ejection fraction (40-50\%). Eur $J$ Heart Fail 2014;16:1049-55.

27. Chen ZM, Jiang LX, Chen YP, et al. Addition of clopidogrel to aspirin in 45,852 patients with acute myocardial infarction: randomised placebo-controlled trial. Lancet 2005;366:1607-21.

28. Baigent C, Landray MJ, Reith C, et al. The effects of lowering LDL cholesterol with simvastatin plus ezetimibe in patients with chronic kidney disease (Study of Heart and Renal Protection): a randomised placebo-controlled trial. Lancet 2011;377:2181-92.

29. HPS2-THRIVE Collaborative Group. HPS2-THRIVE randomized placebo-controlled trial in 25673 high-risk patients of ER niacin/laropiprant: trial design, pre-specified muscle and liver outcomes, and reasons for stopping study treatment. Eur Heart $J$ 2013;34:1279-91.

30. Landray MJ, Haynes R, Hopewell JC, et al. Effects of extendedrelease niacin with laropiprant in high-risk patients. N Engl J Med 2014;371:203-12. 